\title{
Distributed Uplink Signal Processing of Cooperating Base Stations based on IQ Sample Exchange
}

\author{
Christian Hoymann \\ Ericsson Research \\ Aachen, Germany
}

\author{
Laetitia Falconetti \\ Ericsson Research \\ Aachen, Germany
}

\author{
Rohit Gupta \\ University of Washington \\ Seattle, Washington, USA
}

\begin{abstract}
Cellular systems in general suffer from co-channel interference, when simultaneous transmissions in other cells use the same physical resources. In order to mitigate such co-channel interference cooperating Base Stations (BSs) can perform joint multi-antenna signal processing across cell borders.

This paper describes a concept of distributed cooperation, where BSs communicate directly via a BS-BS interface without central control. A serving BS can serve its terminals on its own or it can request cooperation from one or more supporting BSs. By collecting IQ samples from the supporting BSs' antenna elements, the serving BS can virtually increase its number of receive antennas. Exchanging additional parameters allows applying advanced receiver algorithms, e.g., interference rejection or cancelation. Performance evaluations by means of simulation show the capability of BS cooperation applied to 3GPP LTE in terms of cell and user throughput but it also shows the tradeoff in terms of increased backhaul requirement due to BS-BS communication.
\end{abstract}

\section{INTRODUCTION}

Cellular systems with tight frequency reuse and dense deployment of nodes tend to be interference-limited [1]. In conventional cellular systems, co-channel interference is reduced by radio resource management such as power control, frequency reuse, spreading code assignments, and inter-cell interference coordination.

Multi-antenna reception at a Base Station (BS) allows mitigating interference and increasing carrier signal strength by means of multi-antenna baseband processing. Advanced algorithms, such as interference rejection combining requires channel knowledge, while more advanced algorithms, such as interference cancelation receivers, require decoding of interfering data streams [1]. In theory, one can perfectly cancel co-channel interference if interference is known at the receiver and if the number of receive antennas is larger than the number of interferers. Perfect cancelation leads to noninterfered transmissions with a capacity comparable to the Shannon capacity of AWGN channels.

However, channel state information of co-channel User Equipments (UEs) is, in general, not available and co-channel streams are not decodeable due to bad signal quality and due to unknown transmission parameters. The number of antenna elements per BS is limited, and multi-antenna reception at

This work has been partly funded by the German Federal Ministry of Education and Research (Bundesministerium für Bildung und Forschung, BMBF) under the grant 01BU0634. The authors are responsible for the content of this publication. a single BS does not leverage the information available at neighbor BSs. Leveraging antennas of several cells allows multi-user detection and joint transmission across cell borders leading to excellent link quality [2].

Cooperating BSs are often modeled as a single super-BS with more antennas. By contrast, the concept presented in this paper is to mitigate co-channel interference by means of distributed multi-antenna signal processing at multiple cooperating BSs. A given BS, which itself has too few antennas to mitigate co-channel interference sufficiently, collects information from co-channel BS antennas in order to process the signals from a larger number of antennas. Such BSs virtually increase the number of receive antennas. Each BS can request cooperation on-demand: a serving BS can virtually increase its number of receive antennas in case of high co-channel interference and it can process signals of its own antenna elements in case of low co-channel interference.

Distributed cooperation of BSs requires an appropriate BSto-BS interface. It does not affect the core network. For Uplink (UL), required changes to the radio interface are expected to be minor. Thus, UL cooperation of BSs can, e.g., be integrated in the 3GPP logical LTE/SAE architecture. The approach is backward compatible and can be seen as a potential evolution path.

BS cooperation is expected to improve spectral efficiency more in UL than in Downlink (DL). By detecting UE reference symbols, UL channel knowledge is available at the BSs. Due to the limited UE transmission power the UL of mobile systems is, in general, more challenging than the DL. Due to the possible integration of UL cooperation between BS into the 3GPP architecture and its backward compatibility it is expected to be smoothly incorporated in existing operators' networks.

The paper is organized as follows. The concept of BS cooperation in UL is described in section II and is exemplified using Long-Term Evolution (LTE). Section III discusses some BS algorithms affected by cooperation, whereas section IV focusses on the integration of BS cooperation into the conventional cellular system architecture. Finally, some performance results are provided in section $\mathrm{V}$ and section VI concludes the paper. 


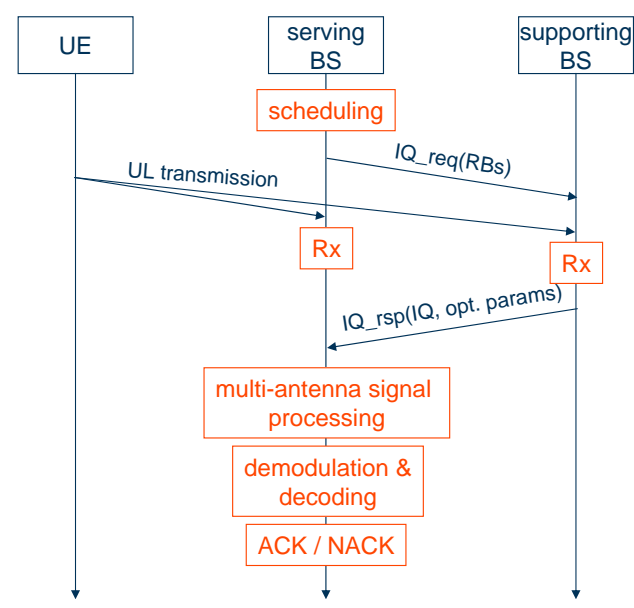

Fig. 1. MSC of requesting IQ samples from a supporting BS

\section{UL COOPERATION OF BASE STATIONS}

A given UE is associated to one serving BS, which controls the UE. During scheduling, the BS allocates certain Resource Blocks (RBs) for UL transmission to the UE. The serving BS can then request support from one (or more) BS for a particular UE transmitting on certain RBs. Figure 1 shows the Message Sequence Chart (MSC) of the cooperation process. Having received the UE signal on the indicated RBs, the supporting BS transfers IQ samples received on its antennas to the serving BS. An IQ sample is the complex representation of a constellation point of a given subcarrier received on a given antenna. It is the output of the Fast Fourier Transform (FFT) at the OFDM receiver chain and basically contains the amplitude and phase with which a particular subcarrier has been modulated, see Fig. 2. Having received IQ samples from the supporting BS, the serving BS jointly processes the received signals of all antennas.

Figure 1 shows that there is no need for a dedicated control node. Whenever the serving BS requires support, it requests it from one or more BS of choice. There are several ways to select an appropriate supporting BS. It can be based on location, on pathloss (long-term channel statistics) or on actual channel realization (short-term), see section III-A.

An example LTE user plane protocol stack of cooperating BSs is shown in Fig. 2. The right hand side of the figure focusses on the LTE BS Physical Layer (PHY) layer. The supporting BS extracts the IQ samples of the indicated RBs from its FFT module and transfers them to the serving BS via the BS-BS interface. This BS-BS interface can be any interface that fulfils the capacity and delay requirements, see section IV. The serving BS exploits the IQ samples in its own PHY layer. Thereby the serving BS's PHY layer virtually increases the number of antenna elements on which the receiver can perform signal processing. Assuming both BSs have four antenna elements each, signal processing is as powerful as if the serving BS would have eight antennas.

Usually, multi-user detection at the serving BS uses receiver algorithms such as Interference Rejection Combining (IRC)

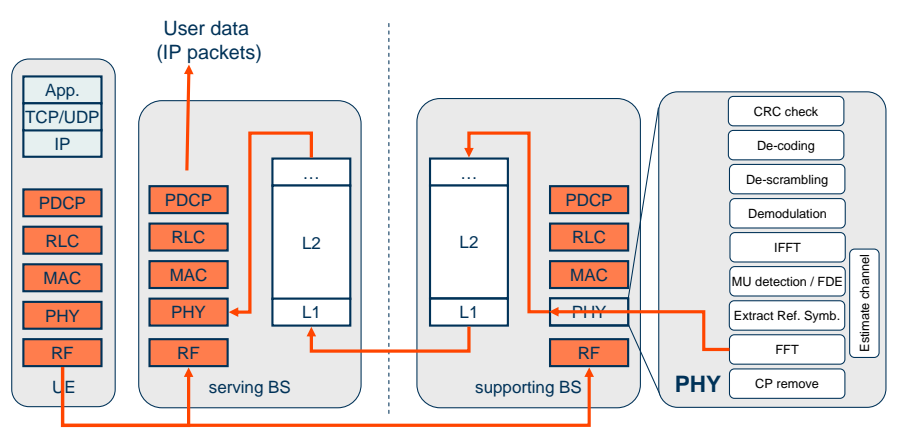

Fig. 2. User plane protocol stack of cooperating LTE BSs

to mitigate co-channel interference. Instead, the serving BS could also apply Interference Cancelation (IC). With IC, the serving BS demodulates and decodes the co-channel streams in order to re-generate the interfering signal. Then the serving BS subtracts the known interference from the received signal. In order to demodulate and decode the interfering streams, the serving BS requires information about the parameters used at the transmitter, i.e., UE. Those parameters can be inserted in the supporting BS's response message (optional parameters in Fig. 1).

\section{AlgorithmS AFFECTED BY BS COOPERATION}

\section{A. Selection of supporting BSs}

By increasing the number of cooperating BSs, the serving BS virtually increases its number of receive antennas. Hence, advanced receiver algorithms can significantly increase Signal to Interference plus Noise Ratio (SINR) of the received signal. Although the serving BS has interest in getting cooperation from many supporting BSs for each UE in its cell, the backhaul capacity puts a restriction on the number of supporting BSs per UE and on the number of UEs that are supported by a BS. As cell edge users are most affected by co-channel interference, they should be prioritized to be supported. Cell edge UEs usually carry out and report co-channel measurements for mobility purposes. These reports can be used by the serving BS to select a set of supporting BSs that will receive a cooperation request.

\section{B. Link adaptation}

In LTE the selection of the Modulation and Coding Scheme (MCS) for the next UL transmission is carried out by the serving BS. Based on the received SINR the serving BS selects the MCS that maximizes the user throughput under the constraint of a Block Error Ratio (BLER) target. BS cooperation increases the UL SINR perceived at the serving BS. Link adaptation should be based on the increased SINR after cooperation. Thereby, the BLER target can be met with a more aggressive MCS, which results in a higher data throughput.

\section{Power control}

The UE transmit (Tx) power consists of an open loop and a closed loop component. In the open loop power control 
mechanism, each UE selects an appropriate Tx power based on the pathloss to the BS on its own. In case of BS cooperation UEs could also consider supporting BSs. Then, UEs need to know which BSs currently cooperate. This reduces the serving BS ability to react quickly to changing transmission conditions.

With closed loop power control the UE Tx power is adjusted by the serving BS by sending a Transmit Power Control (TPC) command. With BSs cooperation, TPC should adjust the UE Tx power to the aggregated receive power of the serving and the supporting BS. Thus, the same quality of service can be achieved with a lower UL Tx power. In this way, BS cooperation enables to decrease the level of co-channel interference.

\section{ARQ mechanisms}

Automatic Repeat Request (ARQ) mechanisms transmit feedback after having received a packet. With BS cooperation, reasonable ACK/NACK feedback can be transmitted by the serving BS only after the joint signal processing has been completed. 3GPP LTE specifies that Hybrid ARQ (HARQ) feedback is sent three Transmission Time Intervals (TTIs) after the data transmission. Thus, either the process of BS cooperation including IQ exchange and joint signal processing is finished in time or the HARQ mechanism needs to be adapted to allow for longer feedback delays.

\section{INTEGRATION OF COOPERATION INTO LTE/SAE LOGICAL ARCHITECTURE}

3GPP's core network (named System Architecture Evolution (SAE)) and the radio access (named Long-Term Evolution (LTE)) are evolving in parallel [3], [4], [5]. The resulting flat architecture is composed of only two logical nodes in the User Plane (UP): the eNodeB and the Serving Gateway (S-GW), see Fig. 3. The S-GW executes packet filtering, classification and it provides the connection to the Internet or to Public Land Mobile Network). An eNodeB provides the LTE radio access. Like in the UP, only two nodes are involved in the Control Plane (CP): the eNodeB and the Mobility Management Entity (MME). The MME handles core network control functions, such as attach/detach handling, mobility functions, bearer management, and security. eNodeBs are connected to the core network using the IP-based interface S1. The logical interface between eNodeBs, i.e., the IP-based X2 interface supports loss-less mobility and multi-cell Radio Resource Management (RRM).

Cooperative BSs with distributed control can be smoothly integrated in the logical architecture since the serving BS keeps controlling the UE. From the core network perspective the serving BS remains the point of contact for both user and control plane. From a UE perspective UL cooperation is transparent, meaning that UEs are not aware whether they are served cooperatively or not. No new node is introduced.

For UL cooperation, a BS-BS interface is required to exchange IQ data between BSs. If BSs of one eNodeB cooperate the required interface could be eNodeB internal. If

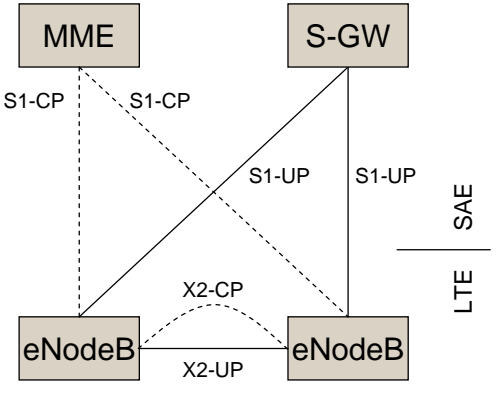

Fig. 3. LTE/SAE logical architecture

BSs of different eNodeBs cooperate the required information is exchanged via the $\mathrm{X} 2$ interface, whose specification would have to be enhanced.

\section{Performance Evaluation}

\section{A. Simulation environment}

The behavior of cooperative BSs has been implemented in a multi-cell radio network simulator modeling OFDM transmission with multi-antenna transmitters and receivers.

In the following, BS cooperation is evaluated in a fully loaded $10 \mathrm{MHz}$ FDD LTE network which consists of 7 sites with three sectors (cells) per site. The inter-site distance is $500 \mathrm{~m}$. Each cell has 10 users in average and operates at a carrier frequency of $2 \mathrm{GHz}$. BSs have an IRC receiver. The evaluation assumptions are essentially based on the Next Generation Mobile Networks (NGMN) recommendations [6]. The channel model used for evaluation is the urban scenario outlined in [7].

In a $10 \mathrm{MHz}$ LTE system, 50 resource blocks are available for UL transmission. A channel dependent BS scheduler allocates a group of 5 resource blocks to 10 users according to their experienced channel conditions. If there are more than 10 users in a cell, the scheduler selects in a first step the users that have been scheduled least previously. If there are less than 10 users in a cell, some resource blocks are left empty.

A conventional open loop power control, which is not adapted to BS cooperation, is applied. Link adaptation allows QPSK, 16QAM, and 64QAM modulation schemes. Turbo coding with adaptive rate matching allows for various combinations of MCSs. Link adaptation operates ideally on the increased SINR after BS cooperation. The MCS for the current transmission is selected based on perfect knowledge of the experienced SINR at the receiver. A link-to-system interface based on mutual information [8] maps the packet SINR to the corresponding BLER. The extra delay introduced by BS cooperation is assumed to be low enough so that HARQ feedback can be send in time.

The maximum number of supporting BSs per UE and the maximum number of UEs supported by a single BS are limited. Note that, in the following, the expression BS is used as if a BS serves only one cell (or sector). For instance, when a serving BS cooperates with 3 supporting BSs, the UE signal is received in 4 different cells. Another parameter 


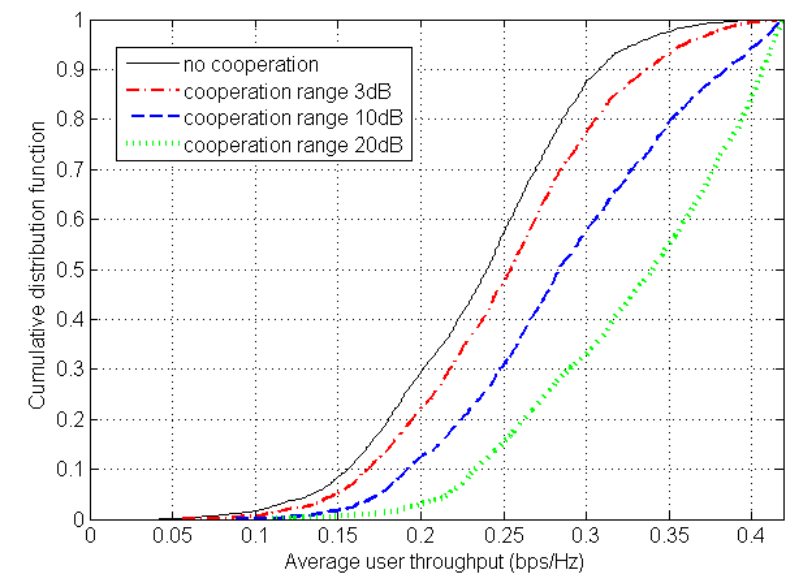

Fig. 4. CDF of average user throughput for different cooperation ranges (max. of 3 supporting BSs)

TABLE I

AVERAGE CELL THROUGHPUT (TP) AND 5\%-TILE USER TP FOR DIFFERENT COOPERATION PARAMETERS

\begin{tabular}{|c|c|c|c|c|}
\hline & \multicolumn{2}{|c|}{ Av. cell TP } & \multicolumn{2}{c|}{ 5\%-tile user TP } \\
\hline & {$[$ bps $/ \mathrm{Hz}]$} & {$[\%]$} & {$[$ bps/Hz] } & {$[\%]$} \\
\hline No cooperation & 2.35 & & 0.134 & \\
\hline 3 supp. BSs, 3dB range & 2.52 & +7 & 0.150 & +12 \\
\hline 3 supp. BSs, 10dB range & 2.87 & +22 & 0.174 & +30 \\
\hline 3 supp. BSs, 20dB range & 3.29 & +40 & 0.217 & +62 \\
\hline 1 supp. BS, 10dB range & 2.67 & +14 & 0.161 & +20 \\
\hline 5 supp. BSs, 10dB range & 2.91 & +24 & 0.175 & +31 \\
\hline
\end{tabular}

called cooperation range enables to determine cell edge UEs. Only UEs measuring a signal strength of co-channel BS within a certain range below the signal strength of the serving cell are chosen for cooperation. In the following the impact of the maximum number of supporting BSs per UE and the cooperation range on performance is studied.

\section{B. Performance evaluation}

Figure 4 shows the enhancement in the average user throughput due to cooperative BSs. High rate as well as low rate users benefit. When increasing the cooperation range from 3 to $10 \mathrm{~dB}$ a higher number of potentially supporting BSs can be received by the UEs within that range. Thus the number of UEs eligible for cooperation as well as the number of supporting BSs per UE increases.

Table I shows the average cell throughput and the five percentile user throughput obtained for different parameters. The average cell throughput is increased by about $22 \%$ with BS cooperation within $10 \mathrm{~dB}$ range with up to 3 supporting BSs. As expected a larger gain is observable for the low rate users. The five percentile point is improved by $30 \%$. With an increased cooperation range of $20 \mathrm{~dB}$ the average cell throughput increases by $40 \%$ and the five percentile point increases by $62 \%$.

Figure 5 shows the Cumulative Distribution Function (CDF) of the average number of supporting BSs per UE. $35 \%$ of all

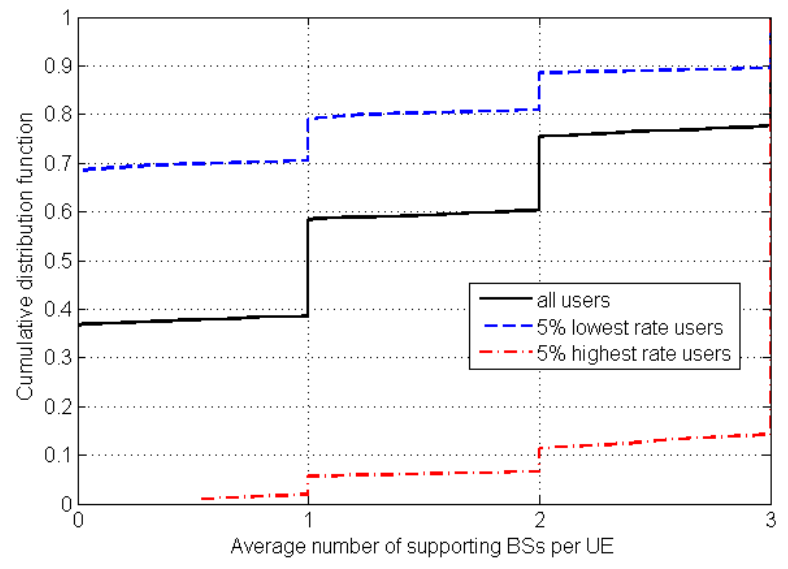

Fig. 5. CDF of average number of cooperative BSs per UE (max. of 3 supporting BSs, cooperation range of $10 \mathrm{~dB}$ )

UEs do not get support from cooperating BSs at all. About $20 \%$ of all UEs are supported by the maximum of 3 BSs. All users achieving highest throughput are supported by one or more BSs: $75 \%$ are supported by 3 BSs. By contrast, users achieving the lowest throughput are supported less by BS cooperation: About $70 \%$ get no support at all and only $10 \%$ are supported by 3 BSs.

Cell edge users usually receive signals from a number of co-channel BSs within the cooperation range so they are supported by co-channel BSs. Cell center users are usually not supported because they do not receive signal from other BSs within the cooperation range. However, Fig. 5 indicates that the group of supported users perceive an unproportionately high throughput, meaning that the throughput of celledge users has exceeded the average throughput. Hence, by means of BS cooperation one could fundamentally modify the distribution of perceived capacity within the cell area, which usually decreases towards the cell edge. An equal and fair distribution all across the cell area can be reached.

Figure 6 shows the impact of the maximum number of supporting BSs per UE. If a serving BS is allowed to cooperate with more supporting BSs it can exploit signals received by more supporting BS antennas. Depending on the quality of the received signals, the SINR of the UE can be further improved and a higher order MCS can be used, resulting in a user throughput rise.

When increasing the number of supporting BSs from 1 to 3 , especially high rate users benefit. Those users are already supported by cooperating BSs, see Fig. 5, however, now they can be supported by even more BSs. Low rate users do not benefit much. They do not receive many BSs within the cooperation range anyway so they cannot increase the number of supporting BSs.

Increasing the maximum number of cooperative BSs per UE further from 3 to 5 does not enhance the user throughput much. The cooperation range of $10 \mathrm{~dB}$ limits the increase in supporting BSs. The $4^{\text {th }}$ and $5^{\text {th }}$ candidate BSs are simply 


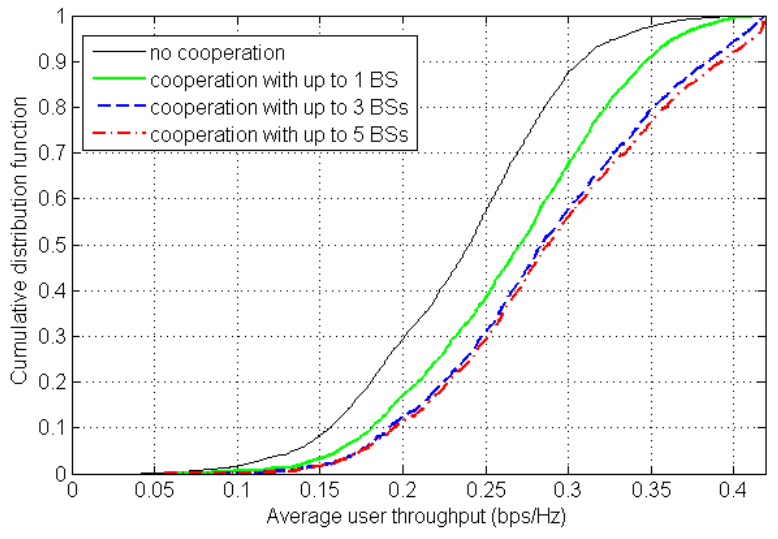

Fig. 6. CDF of average user throughput for different maximum numbers of supporting BSs (cooperation range of $10 \mathrm{~dB}$ )

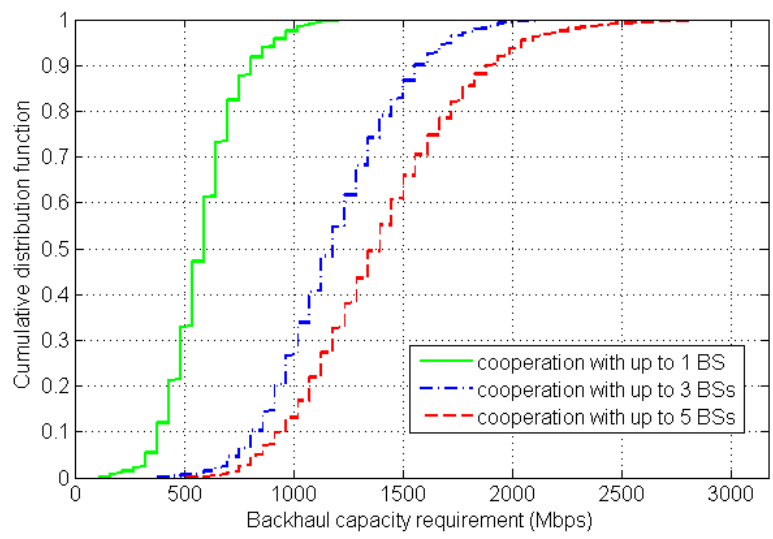

Fig. 7. CDF of backhaul capacity requirement for different values of the maximum number of cooperative BSs (cooperation range of 10dB)

too far away from the UE so that they do not receive the UE signal reasonably strong.

\section{Backhaul requirement}

The required backhaul capacity is measured on each of the $\mathrm{BSs}$ as the sum of the input and output traffic generated due to the exchange of I/Q vectors. One I/Q sample is assumed to be quantized with 16 bit. Figure 7 shows the resulting backhaul requirement for different numbers of cooperating $\mathrm{BSs}$ for a given cooperation range of $10 \mathrm{~dB}$. Cooperating with only 1 supporting BS results in an average backhaul capacity of $600 \mathrm{Mbps}$. The required average backhaul capacity increases to $1200 \mathrm{Mbps}$ and $1400 \mathrm{Mbps}$ when cooperating with 3 and 5 $\mathrm{BSs}$, respectively.

Obviously, increasing the number of cooperating BSs increases the backhaul requirement, however, user throughput is increased as well. The optimum point in configuring the number of co-operating BS depends on the operator's core network capacity. In order to support the operators' decision, a new metric can be introduced: how much average backhaul capacity do I need to spend in order to increase average cell throughput? Table II answers that question.
TABLE II

REQUIRED BACKHAUL CAPACITY PER INCREASED CELL THROUGHPUT

\begin{tabular}{|c|c|}
\hline & $\begin{array}{c}\text { required backhaul capacity [bps] per } \\
\text { increased cell throughput [bps] }\end{array}$ \\
\hline 1 supporting BS, 3dB range & 9 \\
\hline 3 supporting BSs, 3dB range & 11 \\
\hline 1 supporting BS , 10dB range & 22 \\
\hline 3 supporting BSs, 10dB range & 41 \\
\hline 5 supporting BSs, 10dB range & 48 \\
\hline
\end{tabular}

\section{CONCLUSION}

Cooperative BSs allow mitigating co-channel interference and increasing received signal strength by means of distributed multi-antenna signal processing, an effective and highly efficient method. The number of antenna elements with which a serving BS jointly processes received signals can be virtually increased. The maximum number of elements is only limited by the transmission capability of the BS-BS interface and the processing power at the serving $\mathrm{BS}$.

Performance evaluation by means of simulation showed the great capability of BS cooperation applied to LTE: the average cell throughput could be increased between 7 and 40\%; the 5\%-tile user throughput increased between 12 and 62\%. As a trade-off, the requirement on backhaul capacity increases, too: between 600 and $1400 \mathrm{Mbps}$ are required for the information exchange between BSs.

Finally, cooperation can be integrated in the 3GPP logical LTE/SAE architecture either as an intra-eNodeB or as an inter-eNodeB feature. The approach is implicitly backward compatible and can be seen as a potential evolution of cellular LTE systems.

\section{REFERENCES}

[1] J. Andrews, "Interference cancellation for cellular systems: a contemporary overview," IEEE Wireless Communications, pp. 19-29, Apr 2005.

[2] P. Marsch and G. Fettweis, "A framework for optimizing the uplink performance of distributed antenna systems under a constrained backhaul," in Proceedings of the IEEE International Conference on Communications ICC, 2007.

[3] 3GPP, “3GPP TS 36.300, Technical Specification Group Radio Access Network; E-UTRA and E-UTRAN; Overall description,” Sep 2006.

[4] — "3GPP TS 23.401, Technical Specification Group Services and System Aspects; GPRS enhancements for E-UTRAN access," Mar 2008.

[5] E. Dahlman, S. Parkvall, J. Skold, and P. Beming, $3 G$ Evolution: HSPA and LTE for Mobile Broadband. Academic Press, August 2007.

[6] NGMN, "NGMN Radio Access Performance Evaluation Methodology," June 2008.

[7] 3GPP, "3GPP TR 25.996, Technical Specification Group Radio Access Network; Spacial channel model for Multiple Input Multiple Output simulations," June 2007.

[8] K. Brueninghaus, D. Astely, T. Salzer, S. Visuri, A. Alexiou, S. Karger, and G. Seraji, "Link performance models for system level simulations of broadband radio access systems," IEEE 16th International Symposium on Personal, Indoor and Mobile Radio Communications, 2005. PIMRC 2005, vol. 4, 2005. 


$\begin{array}{ll}\text { 3GPP } & \text { 3rd Generation Partnership Project } \\ \text { ARQ } & \text { Automatic Repeat Request } \\ \text { AWGN } & \text { Additive White Gaussian Noise } \\ \text { BLER } & \text { Block Error Ratio } \\ \text { BS } & \text { Base Station } \\ \text { CDF } & \text { Cumulative Distribution Function } \\ \text { CP } & \text { Cyclic Prefix } \\ \text { CP } & \text { Control Plane } \\ \text { DL } & \text { Downlink } \\ \text { FFT } & \text { Fast Fourier Transform } \\ \text { HARQ } & \text { Hybrid ARQ } \\ \text { IC } & \text { Interference Cancelation } \\ \text { IRC } & \text { Interference Rejection Combining } \\ \text { LTE } & \text { Long-Term Evolution } \\ \text { MCS } & \text { Modulation and Coding Scheme } \\ \text { MME } & \text { Mobility Management Entity } \\ \text { MSC } & \text { Message Sequence Chart } \\ \text { NGMN } & \text { Next Generation Mobile Networks } \\ & \\ \text { PHY } & \text { Physical Layer } \\ \text { PLMN } & \text { Public Land Mobile Network } \\ \text { RB } & \text { Resource Block } \\ \text { RRM } & \text { Radio Resource Management } \\ \text { SAE } & \text { System Architecture Evolution } \\ \text { S-GW } & \text { Serving Gateway } \\ \text { SINR } & \text { Signal to Interference plus Noise Ratio } \\ & \\ \text { TPC } & \text { Transmit Power Control } \\ \text { TTI } & \text { Transmission Time Interval } \\ \text { Tx } & \text { transmit } \\ \text { UE } & \text { User Equipment } \\ \text { UL } & \text { Uplink } \\ \text { UP } & \text { User Plane } \\ & \\ & \end{array}$

